\title{
Nanomedicines for cancer therapy: current status, challenges and future prospects
}

\begin{abstract}
The emergence of nanomedicine as an innovative and promising alternative technology shows many advantages over conventional cancer therapies and provides new opportunities for early detection, improved treatment, and diagnosis of cancer. Despite the cancer nanomedicines' capability of delivering chemotherapeutic agents while providing lower systemic toxicity, it is paramount to consider the cancer complexity and dynamics for bridging the translational bench-to-bedside gap. It is important to conduct appropriate investigations for exploiting the tumor microenvironment, and achieving a more comprehensive understanding of the fundamental biological processes in cancer and their roles in modulating nanoparticle-protein interactions, blood circulation, and tumor penetration. This review provides an overview of the current cancer nanomedicines, the major challenges, and the future opportunities in this research area.
\end{abstract}

Keyword: Biological barriers; Cancer nanomedicines; Chemotherapeutic agents; Drug delivery; Nanoparticles; Tumor microenvironment 